\title{
Aggregation of Cyanine Dye in Bilayer Vesicles of Phospholipids
}

\author{
Casey McCullough, Matthew Heywood, and Hussein Samha \\ Division of Chemistry \\ Southern Utah University \\ 351 W. Center Street, SC 215 \\ Cedar City, Utah 84720 USA
}

Received: April 1, $2005 \quad$ Accepted: September 26, 2005

\begin{abstract}
The effect of phospholipid, 1,2-Dipalmitoyl-sn-glycero-3-Phosphocholine (DPPC) on the spectroscopy of the cyanine dye, 1-ethyl-1'-octadecyl-2,2'-cyanine iodide (PIC-18), has been investigated using UV-Vis spectroscopy. Vesicles of DPPC containing PIC-18 in the molar ratio of 1:3 (dye/phospholipids) were prepared in aqueous solution. J-aggregates of PIC-18 were detected in the bilayer wall of the vesicles. When an aqueous solution of mixed PIC-18/DPPC vesicles is treated with excess DPPC vesicles that are prepared separately, the dye molecules in the mixed vesicles underwent a rapid (aggregate) $)_{\mathrm{n}} \leftrightarrows \mathrm{n}$ (monomer) equilibrium as the appearance of only one isosbestic point in the absorbance of the dye indicates. The equilibrium constant was calculated at room temperature $\left(\mathrm{K}_{\mathrm{eq}}=6.7 \times 10^{-2}\right)$. An aggregation number of 4 was calculated for the dye in the bilayer vesicles.
\end{abstract}

\section{INTRODUCTION}

Cyanine dyes have long held the interest of the scientific community due to their unique optical properties. Of those properties, their ability to form J-aggregates has received the most attention. Jaggregates of a wide variety of dyes and aromatic compounds have been recognized and extensively investigated in different media [1-5]. This particular aggregation is revealed by an intense narrow absorption band that appears "red-shifted" with respect to the absorption of the monomer. It is believed that this band is a result of tightly packed dye molecules, with "head-to-tail" transition dipole arrangements, forming an excitonic state through electron coupling [6].

Sato and coworkers have succeeded in producing J-aggregates of cyanine dyes without long alkyl chains by adsorbing dye molecules at the surface of phospholipid vesicles [7,8]. Their work however was limited to the presence of the acidic phospholipids in the membrane for the binding of the chromophore to the vesicle.

This paper reports the formation of J-aggregates of 1-ethyl-1'-octadecyl-2,2'cyanine iodide (abbreviated as PIC-18), that has a long alkyl group attached to the chromophore, within the bilayer wall of 1,2Dipalmitoyl-sn-glycero-3-Phosphocholine (abbreviated as DPPC) vesicles. Further, the aggregation number of the dye and the equilibrium constant are calculated.

Structural formulas of the phospholipids and the cyanine dye used in this work are presented in Figure 1.

\section{EXPERIMENTAL SECTION}

Bilayer vesicles of DPPC containing cyanine dye, PIC-18, were prepared in aqueous solution by the standard preparation method [9]. According to the preparation procedure, $0.73 \mathrm{mg}$ dye and 7.3 mg DPPC (1:3 mole ratio) were mixed together in $1.0 \mathrm{~mL}$ chloroform solvent in a 50 $\mathrm{mL}$ round bottom flask. After the solution is 


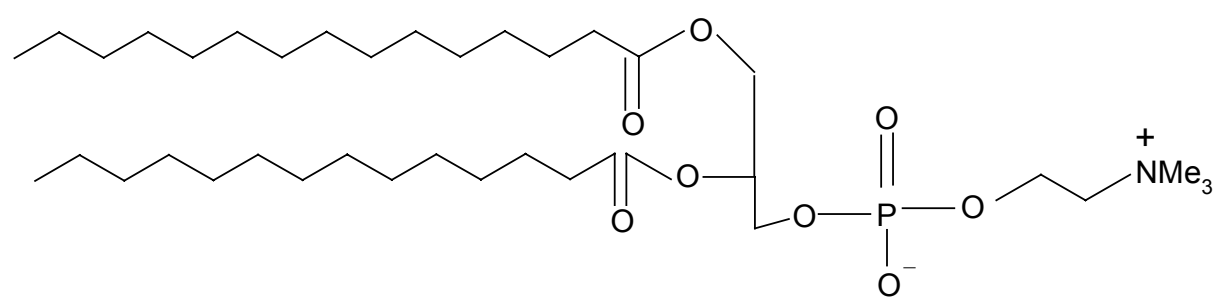

DPPC

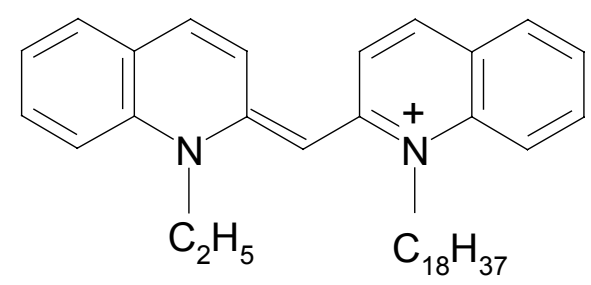

PIC-18

Figure 1. Structural formulas of phospholipid and cyanine dye.

made, the chloroform solvent was evaporated by running pure $\mathrm{N}_{2}$ gas over the solution while manually rotating the flask to create a thin film of the solid mixture of the dye and DPPC on the bottom and sides of the flask. The film was dried under vacuum for 2.0 hours at room temperature. After that, $50 \mathrm{~mL}$ of warm $\left(\sim 50{ }^{\circ} \mathrm{C}\right) \mathrm{H}_{2} \mathrm{O}$ was added to the flask. The flask was agitated to dissolve the thin film in the water, forming a turbid solution. This solution was then sonicated at $47{ }^{\circ} \mathrm{C}$ in a bath sonicator (BRANSON 8200) until the film dissolved completely and a clear solution was obtained (1-2 hrs). For each experiment, $2.0 \mathrm{~mL}$ of the prepared phospholipid-dye solution were placed in a cuvette for absorption measurements. Ocean Optics Spectrophotometer (model CHEM 2000 UVVIS) was used to measure the absorbance of the dye in the solution. After the absorbance of the dye/DPPC vesicles was taken and recorded, $25 \mu \mathrm{L}$ of pure DPPC vesicles, which had been previously prepared by the same procedure above, were added to the cuvette and the solution mixture is mixed quickly by manually shaking the cuvette. Immediately after the mixing, the cuvette was placed in the chamber of the spectrophotometer and the absorbance of the dye was monitored and recorded several times over a period of 1.0 hour. A final measurement was recorded after 24 hours.

\section{RESULTS AND DISCUSSION}

Lipid bilayers form in aqueous solution due to the amphiphilic nature of the phospholipid molecules. Under the right conditions these bilayers form spherical vesicles that separate an interior aqueous environment from the rest of the aqueous solution. In these vesicles, the hydrophobic alkyl chains of the DPPC molecules are pointed toward the inside of the vesicle wall while the hydrophilic heads are facing the aqueous medium on the inside and outside of the vesicle. PIC-18 dye molecules are embedded within the wall of the vesicles by the hydrophobic interaction between the alkyl chains on the dye molecules and the DPPC molecules. A schematic representation of the dye molecules embedded within the bilayer wall of the DPPC vesicles is presented in Figure 2.

The absorbance of the dye in phospholipid vesicles is shown in Figure 3. The spectrum shows the absorbance of the J-aggregate at $578 \mathrm{~nm}$, it also shows some monomer as the shoulder on the left side in the spectrum indicates. The hydrophobicity of the dye, due to the existence of the octadecyl group, makes it very insoluble in 


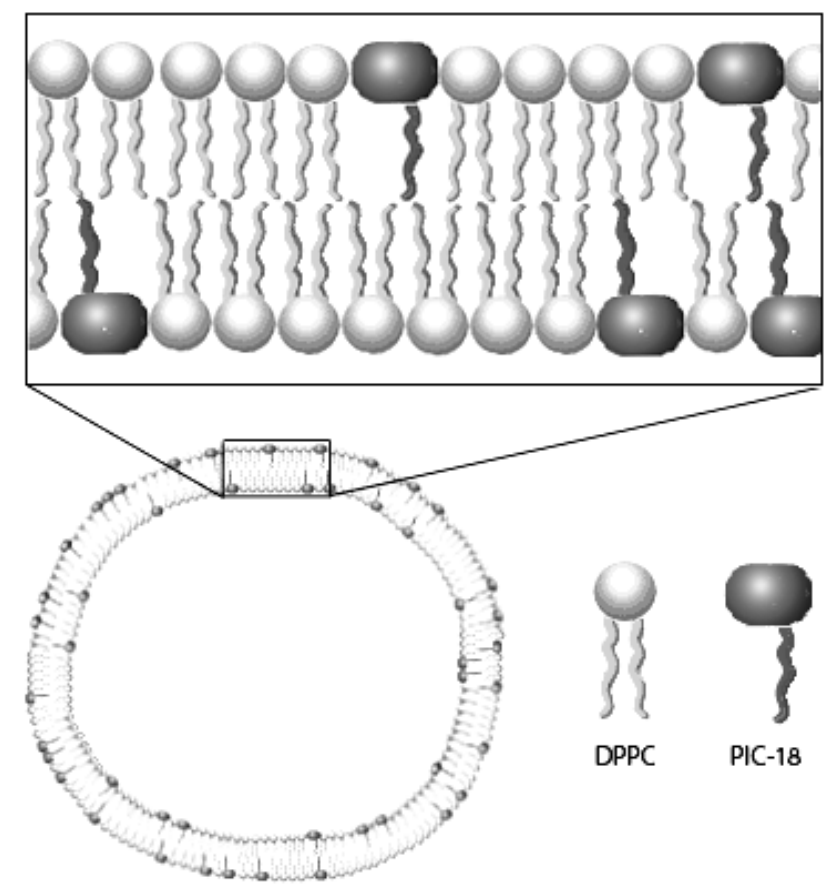

Figure 2. The schematic representation of the dye molecules incorporated within the bilayer wall of the DPPC vesicles.

water. Therefore, dye molecules can only be dragged into aqueous solution by a carrier that matches their structure. In this case, DPPC is utilized to provide a hydrophobic environment for the cyanine dye to dissolve in aqueous solutions. As a result, J-aggregate in the bilayer vesicles are formed as Figure 3 indicates. J-aggregate of PIC-18 was also detected in more dilute mixtures of dye/DPPC. We have examined solutions of mixing ratios of $1: 10,2: 10$, and $3: 10$. In each of these solutions a significant amount of $\mathrm{J}$-aggregate of the dye is formed during sonication. In the 1:10 mixture, J-aggregate continued forming when the solution was kept sitting for several days. Absorption spectrum of the 1:10 mole ratio as it was freshly prepared and after 10 days of preparation is presented in Figure 4. Freshly prepared vesicles show a significant amount of monomeric molecules of the dye that are embedded within the wall of the vesicles as the absorption at $523 \mathrm{~nm}$ in Figure 4 indicates. These monomers are gradually converted to J-aggregate over several days. The growth in the "J-band" in Figure 4 on the expense of the monomer band illustrates the conversion process over a period of 10 days. However, that was not the case for the 1:3 ratio.

The prepared mixed vesicles are stable in aqueous solution and the amount of J-aggregate in the bilayer wall does not change after the equilibrium, aggregate $\leftrightarrows$ monomer, is established. Typically in the case of $1: 3$ mole ratio, this equilibrium is reached during the sonication process. When an aqueous solution of PIC-18/DPPC vesicles is treated with excess of DPPC aqueous solution, the dye molecules underwent a rapid (aggregate) $)_{\mathrm{n}} \leftrightarrows$ $\mathrm{n}$ (monomer) equilibrium. The appearance of only one isosbestic point in the absorption spectra of Figure 5 indicates the equilibrium between the J-aggregate and the monomer. Figure 5 illustrates the change in the absorbance of the dye after the addition of $25 \mu \mathrm{L}$ of pure DPPC vesicles to the mixed PIC-18/DPPC vesicles originally under equilibrium. According to Figure 5, the intensity of the monomer band at $523 \mathrm{~nm}$ is 


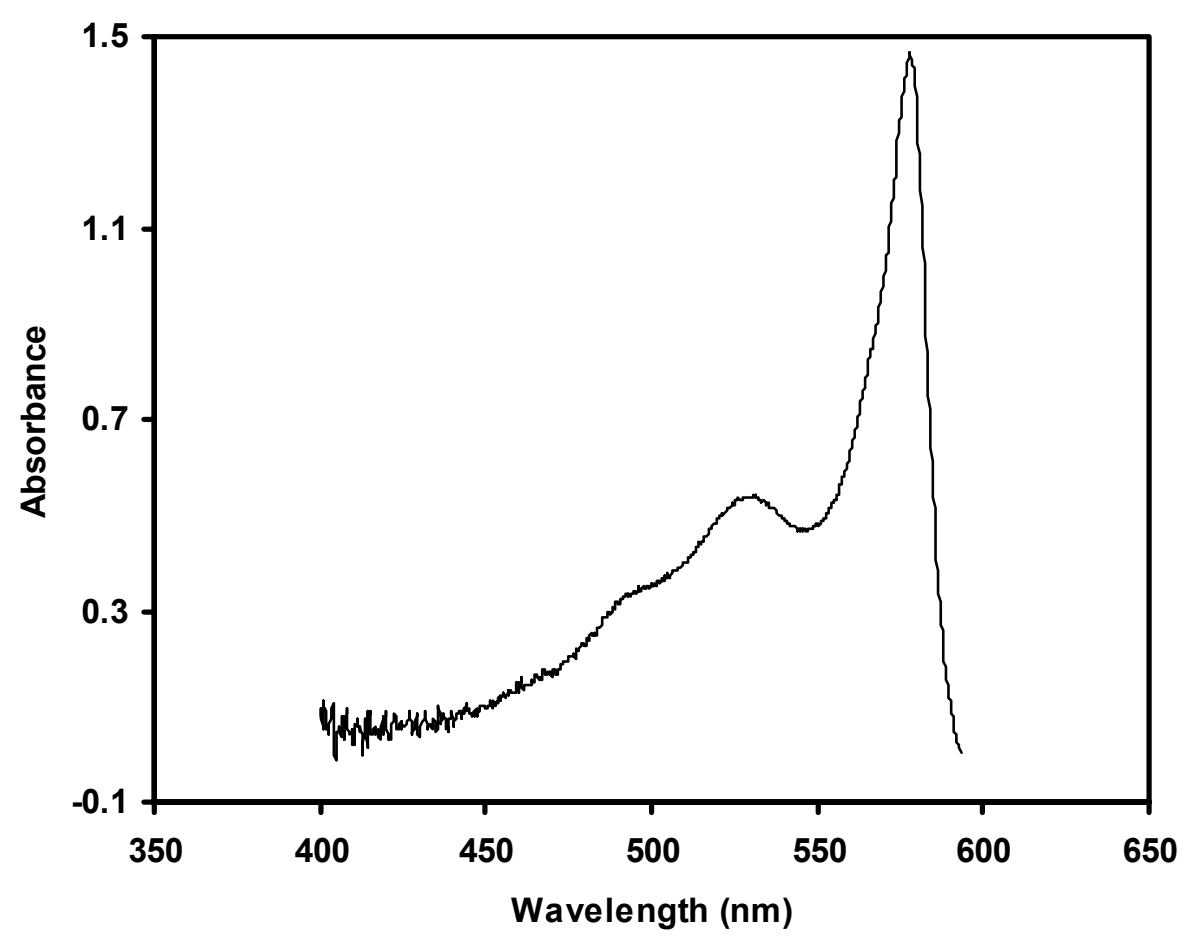

Figure 3. The absorbance of PIC-18 in DPPC vesicles (1:3 mole ratio) in aqueous solution.

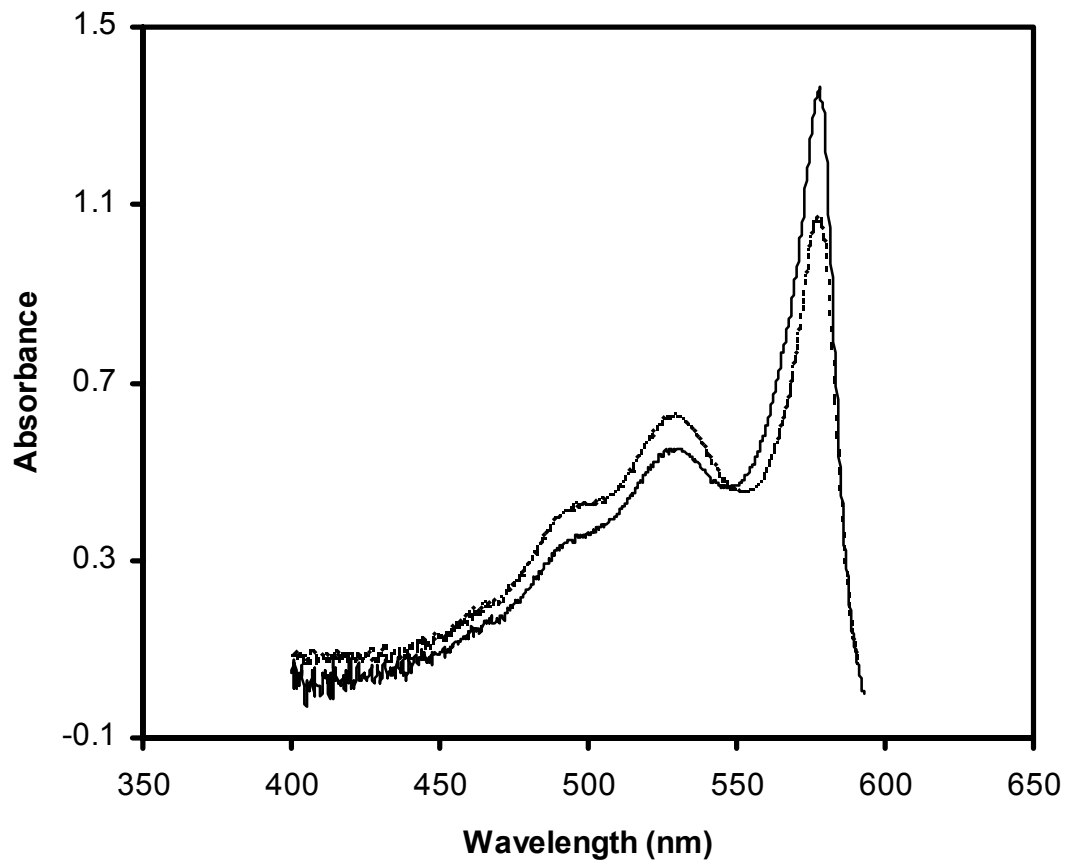

Figure 4. The absorbance of PIC -18 in DPPC vesicles (1:10 mole ratio) (broken line) freshly prepared, (solid line) after 10 days. 


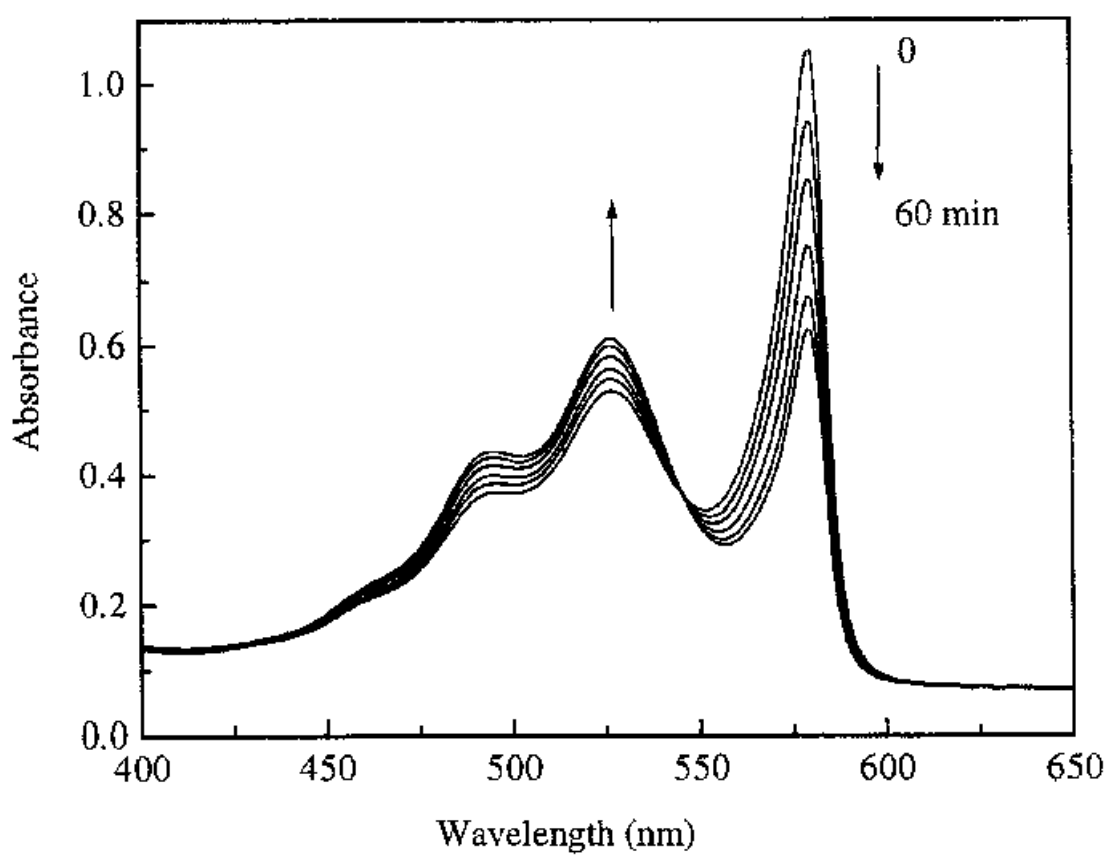

Figure 5. The absorbance of PIC -18 dye in freshly prepared PIC-18/DPPC vesicles (1:3 mole ratio) added to it $25 \mu \mathrm{L}$ of pure DPPC vesicles. Absorbance is recorded every 12 minutes over a period of 60 minutes.

increased at the expense of the $\mathrm{J}$-aggregate band, which decreased. This change in the absorbance is followed upon for a period of 24 hours. We have noticed that the change is significant and noticeable during the first 2 hours. After this period of time a new equilibrium will have been reached at which no further change in the absorbance is noticeable.

The above equilibrium is explained according to equation (1),

$$
\begin{aligned}
& \text { (aggregate })_{\mathrm{n}} \leftrightarrows \mathrm{n}(\text { monomer }) \\
& \mathrm{K}=\frac{[\text { monomer }]^{n}}{[\text { aggregare }]}
\end{aligned}
$$

and

$$
\ln (\mathrm{K})=\mathrm{n} \ln [\text { monomer] }-\operatorname{In} \text { [aggregate], }
$$

where $\mathrm{K}$ is the equilibrium constant. Equation (2) demonstrates the relationship between the aggregate and monomer in vesicles using the aggregation number, $\mathrm{n}$.

According to Benesi-Hildebrand kinetics, the above equilibrium can be described by the change in the absorbance of the J-aggregate, Equation (3), where $A_{\text {agg }}$ and $\mathrm{A}^{\circ}$ agg $-\mathrm{A}_{\text {agg }}$ correspond to the molar concentrations of the aggregate and monomer respectively. Where as $A_{\text {agg, }}^{\circ}$, is the initial absorbance of the J-aggregate before the addition of DPPC while $A_{\text {agg }}$, is the absorbance of J-aggregate at equilibrium.

$$
\ln \left(\mathrm{A}_{\text {agg }}\right)=\mathrm{n} \ln \left(\mathrm{A}_{\text {agg }}^{\circ}-\mathrm{A}_{\text {agg }}\right)+\mathrm{C}
$$

The plot of $\operatorname{In}\left(\mathrm{A}_{\text {agg }}\right)$ versus $\ln \left(\mathrm{A}^{\circ}{ }_{\text {agg }}-\right.$ $A_{\text {agg }}$ ) using absorbance data from three different experiments yields a straight line with a slope of 4, representing the aggregation number $n$ of PIC-18 in DPPC vesicles (Figure 6). $\mathrm{K}_{\mathrm{eq}}=6.7 \times 10^{-2}$ is also calculated from the intersection of the straight line in Figure 6 with the $y$-axis.

\section{CONCLUSION}

A tractable method of producing J-aggregate of cyanine dyes in aqueous solution is reported. The incorporation of PIC-18 in the J-aggregate form within the wall of phospholipid vesicles is potentially important 


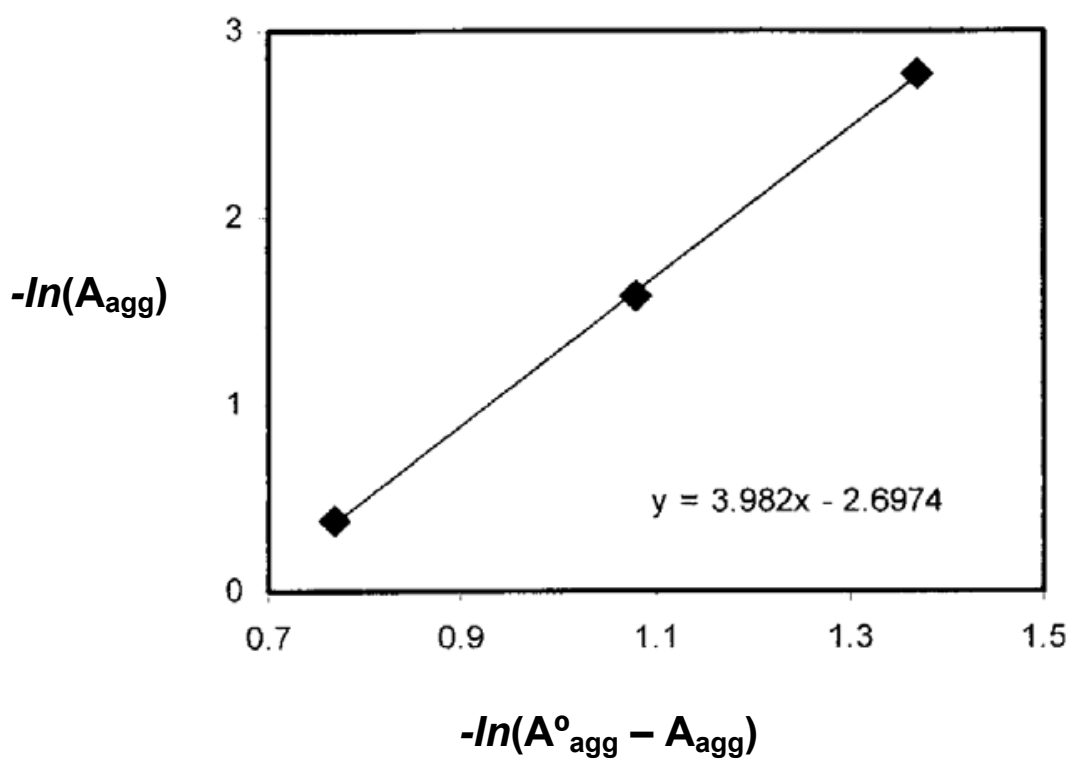

Figure 6. Plot of Henesi-Hildebrand's equation

to better understand the biochemical activities of the cell. The prominent spectroscopic features of J-aggregate introduce the possibility of their use in study of biochemical activities of the cell.

\section{REFERENCES}

1. A. H. Herz, Advances in Colloid and Interface Science, 8, 237 (1977).

2. V. Czikklely; H. D. Forsterling; H. Kuhn, Chem. Phys. Lett., 6, 207 (1970).

3. D. Mobius, Adv. Mater, 7, 437 (1995).

4. W. Tillmann; H. Samha, American Journal of Undergraduate Research, 3(3), 1 (2004).
5. A. Dixon; C. Duncan; H. Samha, American Journal of Undergraduate Research, 3(4), 29 (2005).

6. M. Matsumoto; T. Nakazawa; R. Azumi; H. Tachibana; Y. Yamanaka; H. Sakai; M. Masahiko, J. Phys. Chem. B, 106, 11487 (2002).

7. T. Sato; Y. Yonezawa; H. Hada, J. Phys. Chem. 93, 14 (1989).

8. P. Mustonen; J. A. Virtanen; P. J. Somerharju; P. K. Kinnunen, Biochemistry, 26, 2991 (1987).

9. Avanti Polar Lipids, Inc. Retrieved from www.avantilipids.com/ images/ PreparationOfLiposomes.html, March 23 (2005).

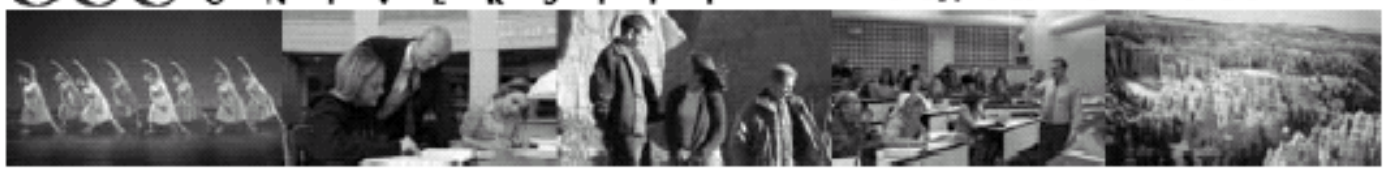

http://www.suu.edu/ 